

\section{ACERCA DE LA AUTORA}

Diana María Robayo-Botiva, magíster en Comercio Electrónico del Instituto Tecnológico y de Estudios Superiores de Monterrey y magíster en gestión de las tecnologías de la información de la Universidad Cooperativa de Colombia. Profesora, Facultad de Ciencias Económicas, Administrativas y Contables, Universidad Cooperativa de Colombia, sede Villavicencio, Colombia.

Correo electrónico: diana.robayo@campusucc.edu.co Google Scholar: https://scholar.google.com/citations? user=XL1poXcAAAAJ\&hl=es

\section{CÓMO CITAR ESTE DOCUMENTO}

Robayo-Botiva, D. M. (2020). El comercio electrónico: concepto, características e importancia en las organizaciones (Generación de contenidos impresos N.20). Bogotá: Ediciones Universidad Cooperativa de Colombia. doi: https://doi.org/10.16925/gclc.13

Este documento puede ser consultado, descargado o reproducido desde nuestro repositorio institucional (http://repository.ucc.edu.co/ handle/20.500.12494/7369) para uso de sus contenidos, bajo la licencia de Creative Commons Reconocimiento-NoComercial-SinObraDerivada 4.0 Internacional. http://creativecommons.org/licenses/by-ncnd/4.0/ 


\title{
20 EL COMERCIO ELECTRÓNICO: E IMPORTANCIA EN LAS ORGANIZACIONES
}

\author{
Diana María Robayo-Botiva
}

\begin{abstract}
Resumen
Internet es una red pública que se ha desarrollado en tres fases desde 1961 al presente. Estos son: la fase de la innovación, en que las ideas básicas y las tecnologías fueron desarrolladas; la fase de la institucionalización, en que esas ideas cobraron vida; y la fase de comercialización, en que finalmente las ideas y las tecnologías fueron implementadas, permitiendo que las compañías privadas llevaran la Internet a millones de personas alrededor del mundo (Laudon y Guercio Traver, 2017). Asimismo, gracias a la fase de comercialización de la Internet, que inició aproximadamente en 1995, se originó el comercio electrónico, que en su primer periodo de desarrollo, fue incipiente debido a la tecnología de banda estrecha utilizada para la conexión a Internet. No obstante, con el surgimiento de la tecnología de banda ancha, además de mejorar los servicios de Internet, también hubo un progreso significativo del comercio electrónico, pasando de ofrecer venta de productos al por menor a vender contenido y servicios. De acuerdo con lo anterior, y teniendo en cuenta la globalización que cada vez cobra mayor importancia a causa de las tecnologías de la información, es fundamental que los estudiantes del área de ciencias económicas, administrativas y contables tengan un conocimiento claro del concepto de comercio electrónico, sus características e importancia dentro de las organizaciones.
\end{abstract}

Palabras clave: comercio electrónico, comercio electrónico parcial, comercio electrónico puro, ventaja competitiva. 


\section{TABLA DE CONTENIDO}

I. INTRODUCCIÓN

II. ¿QUÉ ES COMERCIO ELECTRÓNICO?

III. Diferencia entre COMERCiO y NeGocio Electrónico 5

IV. Características ÚNicAs del comercio eleCtrónico 6

4.1 Ubiquidad 6

4.2 Alcance global 6

4.3 Estándares universales 6

4.4 Riqueza 6

4.5 Interactividad 6

4.6 Densidad de la información $\quad 7$

4.7 Personalización 7

4.8 Tecnología social $\quad 7$

V. TiP os de COMercio electrónico

VI. IMPORTANCIA DEL COMERCIO ELECTRÓNICO EN LAS ORGANIZACIONES 7

VII. CONCLUSIONES

REFERENCIAS $\quad 8$ 


\section{INTRODUCCIÓN}

Esta lectura aborda aspectos básicos del comercio electrónico y su importancia dentro de las organizaciones. Este material está dirigido a estudiantes del área de ciencias económicas, administrativas y contables, e incluye temas como el concepto de comercio electrónico, su diferencia con el negocio electrónico, sus características únicas, sus tipos de acuerdo con el proceso de compra y la ventaja competitiva que pueden alcanzar las organizaciones al implementarlo. A partir de la lectura, los estudiantes reconocerán el valor que ha adquirido el comercio electrónico en las organizaciones en los últimos años debido a la masificación de las tecnologías de la información, que se conciben como el backbone del comercio electrónico.

\section{II. ¿QUÉ ES COMERCIO ELECTRÓNICO?}

A continuación, se presenta el concepto de comercio electrónico desde la perspectiva de tres autores. En primer lugar, el comercio electrónico involucra el uso de Internet, la World Wide Web (WEB), las aplicaciones móviles (apps) y los buscadores (browsers) que corren en los dispositivos móviles para la realización de transacciones de negocio. Aunque los términos Internet y web se intercambian comúnmente, de hecho son muy diferentes. La Internet es una red mundial de computadores, mientras que la WEB es uno de sus servicios más populares, que provee acceso a millones de páginas web. Una app, por su parte, es un software de aplicación. El término es típicamente usado cuando se refiere a aplicaciones móviles, aunque algunas veces se usa para referirse a las aplicaciones de escritorio también. Un browser móvil es una versión de un software de web browser que se accede a través de dispositivos móviles. El comercio electrónico puede ser definido como las transacciones comerciales habilitadas digitalmente entre organizaciones e individuos.
Son transacciones habilitadas digitalmente porque se realizan a través de medios digitales (estas transacciones ocurren en Internet la WEB, en dispositivos móviles) y son transacciones comerciales porque involucran el intercambio de valor (por ejemplo, dinero) entre las organizaciones y los individuos en retorno de un producto o servicio. Algunas veces se refiere al comercio electrónico como comercio digital (Laudon y Guercio Traver, 2017).

En segundo lugar, el comercio electrónico describe el proceso de compra, venta, transferencia, servicio o intercambio de productos y servicios o información mediante una red de computadores, incluyendo Internet (Turban y Volonino, 2010). Finalmente, en tercer lugar, para muchas personas el comercio electrónico significa comprar a través de internet mediante la WEB. Sin embargo, el comercio electrónico incluye otras actividades, tales como comercio entre negocios y procesos internos que las organizaciones usan para soportar la compra, venta, contratación, planeación y otras actividades (Schneider, 2013). De acuerdo con los diferentes conceptos que tienen del comercio electrónico, se puede concluir, en términos generales, que son todas las transacciones comerciales que se realizan a través de una red como Internet, que es la red pública a la cual todas las personas tienen acceso.

\section{DifERENCIA ENTRE COMERCIO Y NEGOCIO ELECTRÓNICO}

Negocio electrónico se refiere a las transacciones y procesos habilitados digitalmente dentro de una organización, involucrando los sistemas de información bajo el control de las organizaciones. Adicionalmente, el negocio electrónico no incluye transacciones comerciales que involucren un intercambio de valor a través de los límites de la organización. Por ejemplo, el mecanismo de control de inventario en línea 
de una organización es un componente del negocio electrónico, pero tales procesos internos no generan directamente ingresos a la organización (Laudon y Guercio Traver, 2017).

De acuerdo con esto, el comercio electrónico esta constituido por transacciones comerciales que se realizan a través de Internet, mientras que el negocio electrónico utiliza las tecnologías de información y los sistemas de información, procurando mejorar procesos y transacciones dentro de las organizaciones. Sin embargo, cabe destacar que tanto para el comercio como para el negocio electrónico la infraestructura tecnológica de una organización es fundamental.

FIGURA 1. Diferencia entre comercio y negocio electrónico. Elaboración propia.

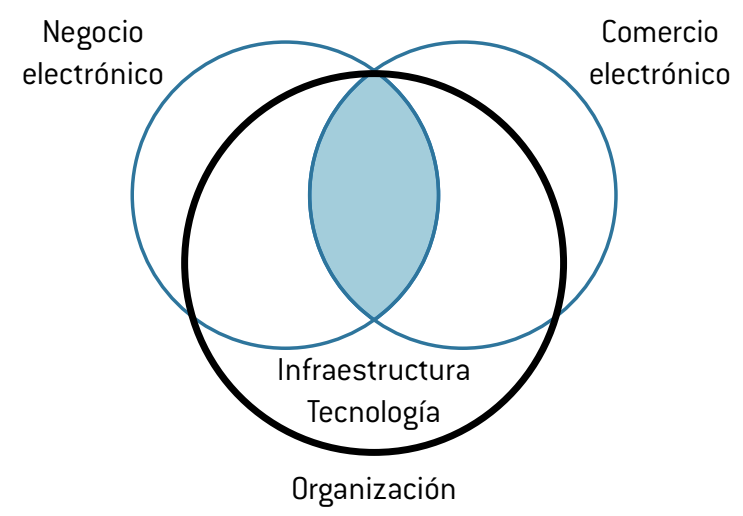

\section{CARACTERÍSTICAS ÚNICAS DEL COMERCIO ELECTRÓNICO}

\section{UBIQUIDAD}

Hace referencia a que el comercio electrónico esta disponible en cualquier lugar y a cualquier hora del día, ya que permite comprar desde un computador, un dispositivo móvil, en la casa, en el carro, o en el lugar en que se encuentre el consumidor. El resultado de esto es un marketspace (espacio del mercado). Esto es, se traspasan los límites tradicionales del espacio y del tiempo. Por tanto, la ubiquidad reduce los costos en las transacciones y la energía cognitiva (Laudon y Guercio Traver, 2017)

\section{ALCANCE GLOBAL}

La tecnología del comercio electrónico permite transacciones comerciales traspasando los límites regionales, nacionales y culturales. El tamaño potencial del mercado para el comercio electrónico es igual al tamaño de la población mundial en línea (Laudon y Guercio Traver, 2017).

\section{ESTÁNDARES UNIVERSALES}

Una característica llamativa del comercio electrónico es que los estándares técnicos de Internet y, por lo tanto, los estándares técnicos que utiliza el comercio electrónico son estándares universales, que son compartidos por todas las naciones alrededor del mundo, los cuales no tienen un costo, solo se deben cumplir. Por tanto, los costos de entrada al mercado son más bajos, igual que los costos de búsqueda. Esto, permite el descubrimiento de precios y externalidades de la red (Laudon y Guercio Traver, 2017).

\section{RIQUEZA}

El comercio electrónico tiene el potencial para ofrecer más riqueza en la información porque permiten la interactividad y ajustar el mensaje de acuerdo con las necesidades del usuario. Un ejemplo de esto es la figura de los asesores virtuales. Adicionalmente, la riqueza habilita la venta de servicios y bienes más complejos, que anteriormente exigían un contacto presencial (como el caso de los servicios financieros y turísticos, por ejemplo) (Laudon y Guercio Traver, 2017)

\section{INTERACTIVIDAD}

Los sitios ofrecen a consumidores espacios como foros y uso de redes sociales con la función de compartir experiencias con los servicios y productos ofertados. Es el caso de los botones de Me gusta y Compartir, que proporcionan a los consumidores la posibilidad de interactuar con el negocio y otros consumidores (Laudon y Guercio Traver, 2017). 


\section{DENSIDAD DE LA INFORMACIÓN}

Es la cantidad total y la calidad de la información disponible para todos los participantes del mercado. Es importante destacar que las tecnologías de la información contribuyen a una constante actualización y precisión de la información. La densidad de la información garantiza la transparencia y la discriminación en los precios (Laudon y Guercio Traver, 2017).

\section{PERSONALIZACIÓN}

En el comercio electrónico, la personalización se puede ver desde dos perspectivas. La primera es la focalización de los mensajes de mercadeo acorde a las necesidades, intereses y compras anteriores de los consumidores. La segunda perspectiva es la posibilidad que tiene el consumidor de personalizar el producto o el servicio basado en las preferencias del mismo (Laudon y Guercio Traver, 2017).

\section{TECNOLOGIÁ SOCIAL}

El comercio electrónico permite a los usuarios crear y compartir contenido con la comunidad del WEB. Usando estas formas de comunicación, los usuarios son capaces de crear nuevas redes sociales y fortalecer las existentes (Laudon y Guercio Traver, 2017). Estos espacios benefician a las organizaciones, pues permiten la interacción entre esta y los consumidores, conociendo sus opiniones y sugerencias, que luego pueden ser utilizados por la organización para mejorar, innovar productos, servicios, entre otros.

\section{Tipos DE COMERCIO ELECTRÓNICO}

El comercio electrónico puede tomar muchas formas dependiendo el grado de digitalización del producto, el proceso de compra/venta y la entrega. Cuando el producto, el proceso de compra/venta y la entrega es digital se habla de comercio electrónico puro (Turban y
Volonino, 2010). Un ejemplo de comercio electrónico puro sería el comprar un libro electrónico, que se paga de manera electrónica y se genera un enlace de descarga.

El comercio electrónico parcial es cuando el producto o el proceso de compra/venta o la entrega no son digitales. Por ejemplo, cuando se compra una camisa en una tienda electrónica, se paga a través de medios digitales y la entrega es a través de una empresa de transporte. Este es un claro ejemplo que ilustra que no todo el proceso es netamente digital.

Cabe mencionar que existen otros tipos de comercio electrónico de acuerdo con los actores que intervienen, como el B2C (comercio electrónico de negocio a consumidor), B2B (comercio electrónico de negocio a negocio), C2C (comercio electrónico de consumidor a consumidor), entre otros, que se espera sean tema de consulta del estudiante.

\section{IMPORTANCIA DEL COMERCIO ELECTRÓNICO EN LAS ORGANIZACIONES}

Las organizaciones invierten en sistemas de información para lograr seis objetivos de negocio estratégicos: excelencia operacional, nuevos productos, servicios y modelos de negocio, intimidad con el cliente y con el proveedor, toma de decisiones mejorada, ventaja competitiva y sobrevivencia (Laudon y Laudon, 2016). Respecto a estos objetivos, se destacan los nuevos modelos de negocio que, con el uso de las tecnologías de la información, permiten que las organizaciones implementen modelos de comercio electrónico como e-tailer, creador de mercado, proveedores de contenido, proveedores de comunidad (modelos B2C), entre otros (Laudon y Guercio Traver, 2017), que llevan a que las organizaciones alcancen un mayor número de clientes a nivel global. 
Adicionalmente, dentro de las funciones fundamentales de los sistemas de información en los negocios esta el apoyo de estrategias para lograr una ventaja competitiva, que se refiere a la aplicación innovadora de las tecnologías de la información en las organizaciones, como por ejemplo la implementación de una aplicación de comercio electrónico para la compra en línea, lo que atraería nuevos clientes y crea la lealtad del cliente, logrando dicha ventaja competitiva en las organizaciones (O'brien y Marakas, 2011).

Lo anterior deja ver que el comercio electrónico en la actualidad es una oportunidad, ya que su implementación permitirá múltiples beneficios como alcanzar un mayor número de clientes debido al alcance global, atraer nuevos clientes, establecer múltiples canales interactivos de comunicación gracias a las tecnologías sociales, proporcionar personalización, ofrecer diferentes medios de pagos (electrónicos y tradiciones), y muchos beneficios más, de ahí parte de su importancia para las organizaciones.

\section{Conclusiones}

El comercio electrónico son las transacciones comerciales que se realizan a través de medios digitales.

El comercio electrónico contempla ocho características únicas.

El comercio y negocio electrónicos son conceptos diferentes, sin embargo, convergen en el uso de una infraestructura tecnológica.

Dependiendo del grado de digitalización del producto, el proceso de compra/venta, y la entrega, se puede presentar comercio electrónico puro o comercio electrónico parcial.

El comercio electrónico ofrece a las organizaciones una ventaja competitiva.

\section{REFERENCIAS}

Laudon, K. y Guercio Traver, C. (2017). E-Commerce. Boston: Pearson.

Laudon, K. y Laudon, J. (2016). Sistemas de Información Gerencial. México: Pearson.

O’brien, J. y Marakas, G. (2011). Management Information Systems. Nueva York: McGraw-Hill.

Schneider, G. (2013). Electronic Commerce. Boston: Cengage Learning.

Turban, E. y Volonino, L. (2010). Information Technology for Management. Nueva York: Wiley. 


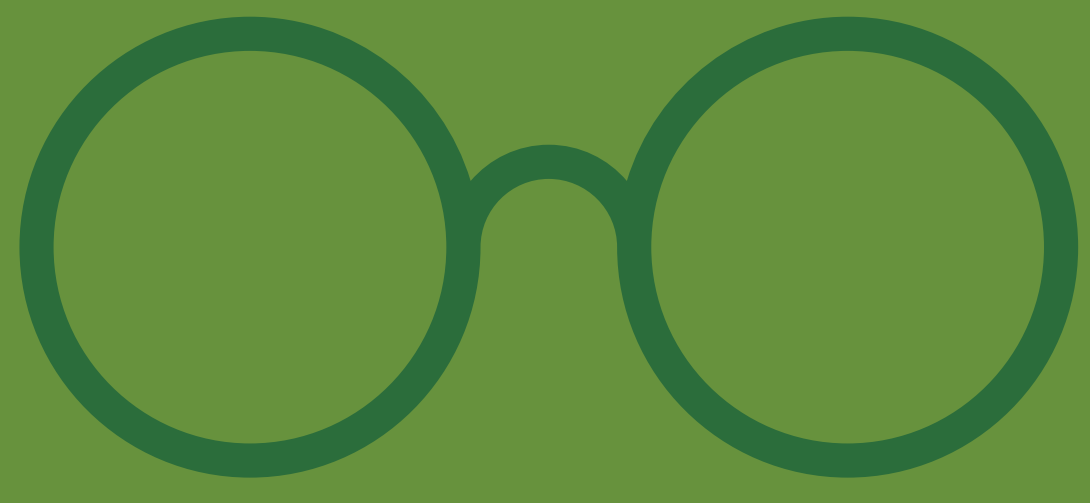

